\title{
Five year follow up of a randomised controlled trial of a stroke rehabilitation unit
}

\author{
N B Lincoln, S Husbands, C Trescoli, A E R Drummond, J R F Gladman, P Berman
}

Caring for stroke patients in stroke units, compared with other hospital locations, leads to decreased mortality and disability at one year. ${ }^{1}$ A trial of a combined acute and rehabilitation ward in Trondheim, Norway, showed that hospital care improved survival and functional outcome at five years. ${ }^{2}$ The benefits were attributed to the treatment provided in the first few weeks after stroke. The purpose of this study was to examine the effects of care in a non-acute stroke unit on outcome at five years.

\section{Methods and results}

The methods and findings of 315 patients at one year of our randomised study comparing a non-acute stroke rehabilitation unit with care on conventional medical and geriatric wards have been published. ${ }^{3}$ In the present study, we ascertained survival to five years by using hospital records and death certificates. Survivors were traced to establish their residential status and visited by a researcher who was blind to group allocation. Subjects were assessed by using all the assessments made earlier, ${ }^{3}$ including the Barthel scale. ${ }^{4}$ Relative risks were calculated for death, death and dependency (using a Barthel score of $\leqslant 18$ to denote dependency), and death and institutionalisation, an institution being defined as a nursing home, residential home, or hospital. Survival curves were generated using the Kaplan-Meier method and compared by using the log rank test.

Altogether 176 patients were randomly allocated to the stroke unit and 139 to conventional wards. The groups were similar at baseline. At five years, 79 (45\%) patients in the stroke unit and $77(55 \%)$ in conventional wards had died, 60 (34\%) patients in the stroke unit and $37(27 \%)$ in conventional wards were disabled (Barthel $\leqslant 18^{4}$ ), and $21(12 \%)$ patients in the stroke unit and $11(8 \%)$ in conventional wards were in institutional care. The relative risks of death $(0.81,95 \%$ confidence interval 0.65 to 1.01 ), death or disability $(0.91,0.83$ to 0.99$)$, and death or institutional care $(0.90,0.75$ to 1.08$)$ were all in the direction of more favourable outcome for patients in the stroke unit. Figure 1 shows the survival curves, which differed significantly (log rank test: $4.36, \mathrm{P}=0.04)$.

Of the 159 patients alive at five years, 139 (90 in the stroke unit) were assessed, and 20 patients (7 in the stroke unit) withdrew from the study. Baseline characteristics of the survivors did not differ between groups, nor did any of the outcomes in terms of personal and instrumental activities of daily living, mood, adjustment, strain on the carer, or mood of the carer (Mann-Whitney U tests $\mathrm{P}>0.05$ ).

\section{Comments}

The long term advantage of stroke units over ordinary care is largely the result of improved survival. Our study was not powered to detect a survival difference,

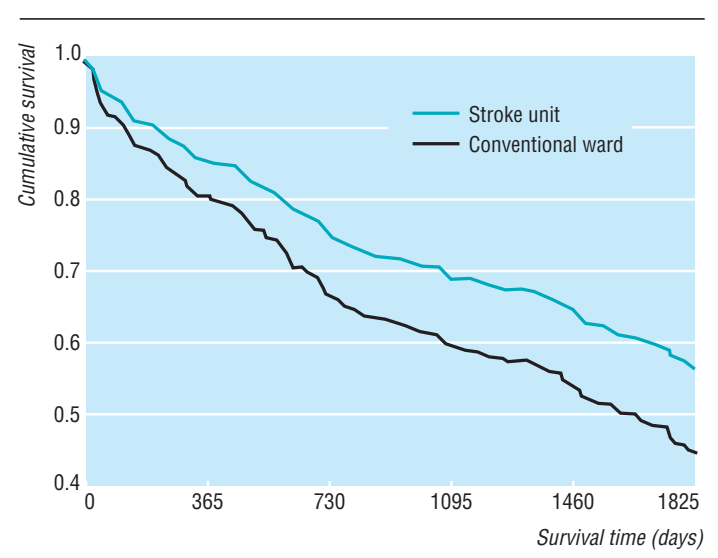

Survival times of patients in stroke unit and in conventional ward (both in Nottingham)

and so our observed survival advantage is imprecise but is similar to that seen in the Trondheim study. ${ }^{2}$ If this estimate were to be confirmed by more studies it would be important for public health.

Mortality differed even though patients in this study were randomised an average of two weeks after their stroke. Our trial does not explain the observed benefits, and we did not monitor medical aftercare. We speculate that improved longer term survival in the stroke unit group is a consequence of the shorter term benefits of the earlier reduction in disability. Differential mortality according to level of disability might also explain why the functional benefit of the stroke unit that was seen up to one year was not seen at five years.

We thank Chris Parker and Elizabeth Atkins for their help with the study, the physicians of City and University Hospitals, Nottingham, for permission to include their patients, and the Stroke Association for financial support.

Contributors: NBL designed and implemented the study and participated in data analysis and writing of the paper. SH assessed outcome with patients, participated in data analysis, and contributed to the writing of the paper. CT ascertained survival, analysed survival data, and contributed to the writing of the paper. AERD was responsible for the design and implementation of the follow-up study, analysis of functional outcomes and contributed to writing the paper.JRFG was responsible for the analysis of survival data and contributed to writing the paper. $\mathrm{PB}$ designed and implemented the study and contributed to the writing of the paper. NBL will act as guarantor for the paper.

Funding: The study was funded by the Stroke Association.

Competing interests: None declared.

1 Stroke Unit Trialists Collaboration. Collaborative systematic review of the randomised trials of organised inpatient (stroke unit) care after stroke. BMJ 1997;314:1151-9.

2 Indredavik B, Slørdahl SA, Bakke F, Rokseth R, Håheim LL. Stroke unit treatment, long-term effects. Stroke 1997;28:1861-6.

3 Juby LC, Lincoln NB, Berman P. The effect of a stroke rehabilitation unit on functional and psychological outcome. Cerebrovasc Dis 1996;6:106-10. 4 Collin C, Wade DT, Davis S, Horne V. The Barthel ADL index: a reliability study. Int Disabil Studies 1988;10:61-3.

(Accepted 7 December 1999)

\section{School of \\ Psychology, University of Nottingham, Nottingham NG7 2RD \\ N B Lincoln professor of clinical psychology Ageing and Disability Research Unit, University of Nottingham, Nottingham NG7 2UH \\ S Husbands research occupational therapist \\ A E R Drummond research officer \\ J R F Gladman senior lecturer in health care of the elderly \\ Department of \\ Medicine, \\ Nottingham City \\ Hospital NHS \\ Trust, Nottingham \\ NG5 1PB \\ C Trescoli senior registrar \\ P Berman consultant physician Correspondence to: N B Lincoln nbl@psychology. nottingham.ac.uk}

BMJ 2000;320:549 